\title{
PD-I and PD-LI as emerging therapeutic targets in gastric cancer: current evidence
}

This article was published in the following Dove Press journal:

Gastrointestinal Cancer:Targets and Therapy

5 May 2017

Number of times this article has been viewed

\author{
Phu N Tran ${ }^{1 *}$ \\ Sarmen Sarkissian ${ }^{*}$ \\ Joseph Chao ${ }^{2}$ \\ Samuel J Klempner ${ }^{3,4}$ \\ 'Division of Hematology-Oncology, \\ University of California Irvine, \\ Orange, ${ }^{2}$ Department of Medical \\ Oncology and Developmental \\ Therapeutics, City of Hope, Duarte, \\ ${ }^{3}$ Samuel Oschin Comprehensive \\ Cancer Institute, Cedars-Sinai \\ Medical Center, ${ }^{4}$ The Angeles Clinic \\ and Research Institute, Los Angeles, \\ CA, USA \\ *These authors contributed equally to \\ this work
}

\begin{abstract}
Gastric adenocarcinoma is a leading cause of global cancer-related morbidity and mortality, and new therapeutic approaches are needed. Despite the improved outcomes with monoclonal antibodies targeting human epidermal growth factor receptor 2 and vascular endothelial growth factor receptor 2, durable responses are uncommon. Targeting immune checkpoints including PD-1, PD-L1 and CTLA-4 have led to improved survival across several tumor types, frequently characterized by prolonged benefit in responding patients. Tumoral and lymphocyte-derived immunohistochemical staining for PD-1, PD-L1, and tumor mutational burden have shown potential as predictive response biomarkers in several tumor types. Optimal incorporation of immune-mediated therapies into gastric cancer (GC) is an area of intense ongoing investigation and benefit has been demonstrated in smaller studies of advanced patients. Important questions of biomarker selection, roles for molecular characterization, optimal combinatorial approaches, and therapeutic sequencing remain. In this study, current data are reviewed for immune checkpoint inhibitors in GC, and putative biomarkers, ongoing trials, and future considerations are discussed.
\end{abstract}

Keywords: immunotherapy, stomach cancer, checkpoint inhibitor, nivolumab, pembrolizumab, tumor mutational burden

\section{Introduction}

Recent global estimates rank gastric cancer (GC) as the fifth most frequently diagnosed cancer (952,000 cases) and the third leading cause of cancer-specific mortality with $\sim 725,000$ deaths worldwide. ${ }^{1}$ There will be 26,370 estimated new cases of GC in the United States in 2016, with an estimated 10,370 deaths, highlighting the high mortality rate. ${ }^{2}$ The 5 -year survival for all stages is $\sim 30 \%$ and $<5 \%$ of stage IV patients are alive at 5 years. ${ }^{2}$ Surgery, radiation, and cytotoxic therapies are the mainstays of locoregional disease, and approval of the biologic agents, trastuzumab and ramucirumab, expanded the armamentarium in advanced GC. The seminal ToGA trial demonstrated the efficacy of incorporating HER2-directed therapy (trastuzumab) in GC patients harboring HER2 overexpression and is now standard in this molecular subgroup. ${ }^{3}$ Ramucirumab, a fully humanized IgG1 monoclonal anti-VEGR2 antibody, is approved in the second line (2L) setting based on improved survival in the REGARD and RAINBOW studies. ${ }^{4,5}$ Absolute improvements remain short with a 2.7-month improvement in median overall survival (OS) for trastuzumab, 1.4 months in the REGARD trial, and 2.2 months in the RAINBOW trials, respectively. ${ }^{4,5}$ Importantly, there are very few durable responses to current therapies, although prolonged
Correspondence: Samuel J Klempner The Angeles Clinic and Research Institute, 11818 Wilshire Boulevard, Los Angeles, CA 90025, USA

$\mathrm{Tel}+\mathrm{I} 31023 \mid 2167$

$\mathrm{Fax}+13102312172$

Email sklempner@theangelesclinic.org 
benefit with trastuzumab in GC with high levels of HER2 amplification has been reported. ${ }^{6}$

The potential of immune-mediated therapies in GC was suggested in early studies with the nonspecific potentiating agents picibanil (OK-432) and polysaccharide-K, an anticancer immunologic adjuvant, dating back to $1975 .{ }^{7}$ More recently, the development of antibodies to immune checkpoints including PD-1, PD-L1, and CTLA-4 have led to a paradigm shift in cancer treatment and the US Food and Drug Administration approvals for nivolumab (Bistol-Myers Squibb; New York, NY, USA), pembrolizumab (Merck\&Co.; Kenilworth, NJ, USA), ipilimumab (Ipi; Bristol-Myers Squibb), and atezolizumab (Genentech; San Francisco, CA, USA) across multiple tumor types. ${ }^{8-18}$ RNA sequencing identified GC as a tumor type associated with local immune cytolytic properties further supporting checkpoint inhibitor investigation. ${ }^{19}$ The present study reviews the current state of immunotherapy in GC with a focus on immune checkpoint inhibitors targeting PD-1 and PD-L1.

\section{Cancer immunity cycle}

Achieving the elimination of tumor cells (TCs) hinges on several key components of the cancer immunity cycle, recognition and stimulation, recruitment, expansion, and ultimately memory. Briefly, TC-derived neoantigens are captured by dendritic cells for processing and presented on major histocompatibility complex class I (MHCI) and class II molecules to T cells, culminating in effector T-cell priming and activation. ${ }^{20}$ The delicate balance of effector and regulatory $\mathrm{T}$ cells determines the nature of the immune response. Once activated, the effector T cells (mainly CD8+) infiltrate the tumor microenvironment and interact with the cancer cells via T-cell receptor (TCR) and its antigen bound to MHCI, leading to cytolytic activity. The dying $\mathrm{TC}$ releases more neoantigens that potentially amplify and propagate further immune response. The binding of the T-cell surface receptor PD-1 to its cognate ligand PD-L1 (B7-H1) or PD-L2 (B7-DC) results in inhibition of T-cell effector function and decreased cytotoxic activity. The ubiquitous TCR CTLA-4 has nonoverlapping suppressive effects on antitumor immunity and is preferentially involved in the earlier immune response, primarily in lymphoid organs. ${ }^{21}$ Understanding the nonoverlapping functions provided rationale for combination therapies in GC and other tumor types, although incorporation of CTLA-4 blockade is generally associated with increased immune-related adverse events (irAEs). Thus far, the greatest therapeutic success has been achieved by interfering with normal checkpoints, specifically the PD-1/PD-L1 axis, commonly co-opted by cancers. ${ }^{20} \mathrm{~A}$ recent preclinical study demonstrated that phosphatidylinositol-4,5-bisphosphate 3-kinase gamma (PI3K $\gamma$ ) is a molecular switch that controls immune suppression. ${ }^{22} \mathrm{PI} 3 \mathrm{~K} \gamma$ inhibition synergized with anti-PD-1 to suppress the growth of human papillomavirus+ (HPV+) head and neck squamous cell carcinoma (HNSCC) tumors and to a lesser extent HPV-HNSCC tumors. ${ }^{22}$ It remains to be seen whether the Epstein-Barr virus (EBV) positive and microsatellite instable (MSI-H) subtype of GC, which have high PIK3CA mutation frequencies, would derive clinical benefits from combined PI3K and PD-1 inhibitors, although there is some isoform specificity with $\mathrm{PI} 3 \mathrm{~K} \gamma$ more commonly involved in immune cells (ICs) over somatic tumor mutations. ${ }^{23}$

\section{GC genomics and immune profiling}

Large-scale molecular characterization of GC by the Asian Cancer Research Group (ACRG) and The Cancer Genome Atlas (TCGA) has highlighted distinct molecular subgroups, with 4 types suggested by the TCGA analyses: EBV-associated, MSI-H, chromosomally instable, and a genomically stable group. ${ }^{23,24}$ The EBV subgroup is associated with amplification of the 9p24.1 locus, which harbors both the $P D-L 1$ and $P D-L 2$ genes. Proteomic studies and messenger RNA (mRNA) analyses confirmed expression of these 2 proteins was highly correlated with genomic amplification. PD-1 expression within tumor-infiltrating lymphocyte cells is observed in more than half of the EBV-positive gastric tumors. ${ }^{25}$ The MSI-high group was associated with high DNA mutation burden as well as DNA hyper-methylation. EBV and MSI comprised $9 \%$ and $22 \%$ of the total, respectively, and immunohistochemical (IHC) studies revealed high PD-L1 staining in association with MSI-high and EBV-positive tumors. ${ }^{25}$ The increased concentration of tumor-infiltrating lymphocytes and expression of PD-L1 provided another surrogate supporting the immunogenicity associated with MSI. ${ }^{24,26}$ Colloquially these have been referred to as "hot" or inflamed tumors while poorly immunogenic tumors are described as "cold." The "hot" tumors, in addition to exhibiting presence of cytotoxic T cells, also strongly express immune-inhibitory pathways, such as PD-L1, indoleamine-2,3-dioxygenase (IDO), and regulatory $\mathrm{T}$ cells. ${ }^{27,28}$ These immune-inhibitory pathways counteract the effects of cytotoxic $T$ cells. In contrast, the "cold" tumors that lack cytotoxic T-cell infiltration do not express immune-inhibitory molecules to the same degree and evade immune destruction partly by T-cell exclusion. 
These tumors do not express important chemokines (ie, CXCL9 and CXCL10) that recruit T cells to the tumor microenvironment. ${ }^{27,29,30}$ Activation of the tumor-intrinsic $\mathrm{Wnt} / \beta$-catenin pathway seems to directly impact T-cell exclusion in melanoma. ${ }^{31}$ The TCGA and ACRG provide a framework for further studies on the intersection of genomics and immunotherapies in $\mathrm{GC}$, and ongoing combinatorial approaches to convert cold tumors to hot may expand the proportion of GC patients for whom immunotherapy may improve outcomes. Furthermore, emerging evidence suggests that there are racial and geographical variations of tumor-immune signatures, which may predict response to immunotherapy. GCs from non-Asian patients were associated with enrichment of tumor-infiltrating lymphocytes and high T-cell gene-expression signatures, such as CTLA-4 signaling. ${ }^{32}$ Increasing routine clinical use of next generation sequencing-based assays which can determine tumor mutational burden (TMB), a presumed surrogate for higher probability of tumor-derived immunogenic neoantigens, may identify those more likely to respond to immune-mediated therapies. ${ }^{33}$ Clinical support for this observation has been demonstrated in MSI (high TMB) tumors, including GC. ${ }^{34,35}$ In urothelial bladder cancer and non-small cell lung cancer (NSCLC) elevated TMB seems to identify a more immuno-responsive subset, although responses are observed in low TMB patients. ${ }^{36-38}$ Whether or not non-MSI elevated TMB will be a predictive response biomarker in GC remains to be determined and requires ongoing clinical trial data sets.

\section{PD-I and PD-LI staining in GC}

Data from NSCLC and other tumor types have suggested that PD-L1 IHC positivity on TCs and/or ICs from biopsy specimens is correlated with predicted benefit from checkpoint inhibitor therapy. ${ }^{39}$ Several series reported IHC positivity rates in $\mathrm{GC}$, and these data are being collected prospectively in ongoing GC immunotherapy trials. ${ }^{40}$ While there are variations in methodologies and antibody clones used, PD-L1 is expressed in up to $65 \%$ of gastric tumors whereas it was undetectable in normal gastric mucosal tissue in healthy subjects..$^{20,25,41,42}$ The frequencies of PD-1 expression in tumor-infiltrating lymphocytes and TCs were $53.8 \%$ and $30.1 \%$, respectively, in another recent series. ${ }^{25}$ There are currently insufficient data to determine if levels of intensity $(<1 \%, 1 \%-24 \%, 25 \%-49 \%,>50 \%)$ or IC to TC scoring systems used in other tumor types can be extended to GC. ${ }^{43}$ Current caveats with the use of PD-L1
IHC include intra-tumoral variability, inter-tumoral variability, temporal variability, and the subjective interpretation of the stains. Different drug developers employ different antibody assays and cutoff values to determine the degree of PD-L1 expression. Each assay includes its own primary antibody, detection system, and scoring criteria, although concordance among the 6 most common antibodies (SP142, E1L3N, 9A11, SP263, 22c3, and 28-8) for PD-L1 detection is high. ${ }^{44}$ The antibody assays for nivolumab, pembrolizumab, avelumab (Merck KgaA; Darmstadt, Germany), and durvalumab (AztraZeneca; Cambridge, UK) are Dako (28-8 Ab), Dako (22c3), Ventana (Merck Clone 73-10), and Ventana (SP263), respectively. ${ }^{45}$ The cutoff thresholds for PD-L1 positivity in most nivolumab studies are 0\%-1\% (negative), 1\%-5\% (weak), 5\%-10\% (medium), and at least $10 \%$ (strong). On the other hand, the cutoff thresholds for pembrolizumab in clinical studies include $0 \%-1 \%$ (negative), 1\%-50\% (medium), and at least 50\% (strong). Consequently, some authors proposed harmonization of PD-L1 testing as an effort to standardize the results. Scheel et al proposed a 6-step scoring system that integrates all cutoff criteria by different antibody assays and found moderate interobserver concordance using this system in PD-L1-positive NSCLC TCs. ${ }^{45}$ Variability in positivity rates and thresholds limit cross-trial comparisons in gastric and other tumor types and ultimately larger prospective data sets and assay harmonization will be important to refine the role of PD-1 and PD-L1 inhibitors in esophagogastric cancers.

There are conflicting reports with regard to PD-L1 expression and prognosis. Some studies reported that patients with PD-L1-positive cancer had significantly shorter survivals compared with those with PD-L1-negative cancer. ${ }^{42,46-48}$ However, recent studies have found PD-L1 expression to be a favorable prognostic marker in GC. ${ }^{25,41,43}$ IC PD-L1 expression was frequently associated with intestinal type cancer, a lower risk of lymph node metastasis, and lower tumor stages compared to MSI-H GCs without PD-L1 expression. ${ }^{41}$ PD-L1 expression in intra- or peritumoral ICs predicts improved survival in patients with high MSI-H gastric carcinoma. ${ }^{41}$ Additionally, higher CD3+ and CD8+ cell density were associated with better OS. ${ }^{49}$ In patients with locally advanced GC receiving adjuvant $S 1$, postoperative neutrophil to lymphocyte ratio (reflected by high neutrophils and low PD-1+ cells) and carcinoembryonic antigen were independent prognostic factors for recurrence after surgery. ${ }^{50}$ Recently, an IHC-based immunoscore from a series of 879 Chinese GC patients suggested that high immunoscore is associated 
with lower recurrence rates and better survival after adjuvant therapy. ${ }^{51}$ It is anticipated that the IHC data collected as part of ongoing trials in locoregional and advanced GC will refine the predictive and prognostic role of PD-1/PD-L1 expression in locoregional and advanced GC.

\section{PD-I and PD-LI checkpoint inhibitors in GC: clinical data}

The promise of improved survival, durable response, and favorable toxicity has spawned a multitude of clinical trials investigating checkpoint inhibitors in GC (Table 1). A nuanced discussion of each trial is beyond the scope of this review, and established data are presented here in the context of other ongoing studies.

\section{Anti-PD-I antibodies}

\section{Nivolumab}

Nivolumab (Bristol-Myers Squibb, New York, NY, USA), like pembrolizumab, is a humanized immunoglobulin G4 monoclonal antibody (mAb) against PD-1 with activity in

Table I Representative clinical trials investigating immunotherapies in gastric and gastroesophageal cancers

\begin{tabular}{|c|c|c|c|c|c|}
\hline Compound & Target or combo & Phase & GC setting & $\begin{array}{l}\text { Primary } \\
\text { endpoint }\end{array}$ & $\begin{array}{l}\text { Clinical } \\
\text { trial ID }\end{array}$ \\
\hline \multicolumn{6}{|l|}{ PD-I monotherapy } \\
\hline JSOOI & PD-I & $\mathrm{lb} / \mathrm{II}$ & $>I$ prior line & ORR & NCT029I5432 \\
\hline Pembrolizumab & PD-I & $1 / I I$ & Neoadj & PCR, PFS & NCT02730546 \\
\hline Pembrolizumab (KEYNOTE-059) & PD-I & II & First-line (cohort 3) & ORR, AEs & NCT023354II \\
\hline Pembrolizumab (KEYNOTE-06I) & PD-I & III & Second-line & OS, PFS in PD-LI+ & NCT02370498 \\
\hline Pembrolizumab (KEYNOTE-062) & PD-I & III & First-line (arm I) & PFS, OS & NCT02494583 \\
\hline Nivolumab & PD-I & III & $>2$ prior lines & OS & NCT02267343 \\
\hline \multicolumn{6}{|l|}{ PD-LI monotherapy } \\
\hline Avelumab (JAVELIN gastric 100 ) & PD-LI & III & First-line maintenance & os & NCT02625610 \\
\hline Avelumab (JAVELIN gastric 300) & PD-LI & III & Third-line & os & NCT02625623 \\
\hline Atezolizumab & PD-LI & 1 & No limits & DLTs & NCTOI 375842 \\
\hline Durvalumab (PLATFORM) & PD-LI & II & $\begin{array}{l}\text { Maintenance after } \\
\text { stage II-III treatment }\end{array}$ & PFS & NCT02678I82 \\
\hline \multicolumn{6}{|l|}{ Dual checkpoint inhibitor } \\
\hline Nivolumab + ipilimumab (CheckMate-649) & PD-I + CTLA-4 & III & First-line & OS & NCT02872116 \\
\hline Durvalumab + tremelimumab & PD-LI + CTLA-4 & $\mathrm{I} / \mathrm{II}$ & $>$ I prior line & AEs, ORR, PFS & NCT02340975 \\
\hline Nivolumab + ipilimumab (FRACTION-GC) & PD-I + CTLA-4 & II & Advanced GC & ORR, DOR, PFS & NCT02935634 \\
\hline \multicolumn{6}{|l|}{ Checkpoint inhibitor + cytotoxic therapy } \\
\hline Pembrolizumab (KEYNOTE-059) & $\mathrm{PD}-\mathrm{I}+\mathrm{Cis} / 5 \mathrm{FU}$ & ॥ & First-line (cohort 2) & ORR, AEs & NCT023354II \\
\hline Pembrolizumab (KEYNOTE-062) & $\mathrm{PD}-\mathrm{I}+\mathrm{Cis} / 5 \mathrm{FU}$ & III & First-line (arm 2) & PFS, OS & NCT02494583 \\
\hline Pembrolizumab & PD-I + FOLFOX & $\mathrm{I} / \mathrm{II}$ & FOLFOX indicated & Safety & NCT02268825 \\
\hline Pembrolizumab & PD-I + chemo & II & Perioperative & DFS at $24 \mathrm{~m}$ & NCT02918162 \\
\hline Pembrolizumab & PD-I + FOLFOX & II & Perioperative & $\mathrm{PCR}, \mathrm{AEs}$ & NCT02943603 \\
\hline Nivolumab & PD-I + SOX or CapOX & II & First-line & ORR & NCT02746796 \\
\hline Pembrolizumab & $\mathrm{PD}-\mathrm{I}+\mathrm{HER} 2+\mathrm{Cis} / 5 \mathrm{FU}$ & $\mathrm{I} / \mathrm{II}$ & First-line & ORR, RP2D & NCT0290I30I \\
\hline \multicolumn{6}{|l|}{ Checkpoint + biologic or other combinations } \\
\hline Pembrolizumab + radiation & PD-I + radiation & ॥ & No limits & Biomarkers & NCT02830594 \\
\hline Ramucirumab + pembrolizumab & VEGFR2 + PD-I & I & 0-2 prior lines & DLTs & NCT02443324 \\
\hline Ramucirumab + durvalumab & PD-LI + VEGFR2 & 1 & I-2 prior lines & DLTs & NCT02572687 \\
\hline $\begin{array}{l}\text { Pembrolizumab with trastuzumab and } \\
\text { cisplatin/5FU }\end{array}$ & PD-I + HER2 + chemo & II & First-line & PFS & NCT02954536 \\
\hline GDC-09 19 and atezolizumab & IDO inhibitor and PD-LI & I & $>$ I prior line & DLTs, AEs & NCT0247I846 \\
\hline Epacadostat and durvalumab & IDO inhibitor + PD-LI & $\mathrm{I} / \mathrm{II}$ & $>$ I prior line & DLTs, ORR & NCT023I8277 \\
\hline PLX3397 and pembrolizumab & CSFIR + PD-I & $\mathrm{I} / \mathrm{II}$ & $>1-2$ prior lines & AEs & NCT02452424 \\
\hline Durvalumab and olaparib (MEDIOLA) & PARP + PD-LI & $\mathrm{I} / \mathrm{II}$ & $>1-2$ prior lines & DCR, safety & NCT02734004 \\
\hline PEGPH20 with pembrolizumab & Hyaluronidase and PD-I & $\mathrm{lb}$ & $>$ I prior line & DLT, ORR & NCT02563548 \\
\hline GS-5745 and nivolumab & MMP-9 and PD-I & II & First-line & ORR & NCT0286438I \\
\hline
\end{tabular}

Notes: Data are derived from clinicaltrials.gov (accessed I/2017). Due to space constraints, a complete list is not shown and preference is given to trials focused on GC and GEJ cancers over those accepting all solid tumors. Several trials have multiple arms; please refer to clinicaltrials.gov for further details.

Abbreviations: VEGFR, vascular endothelial growth factor receptor; MMP, matrix metalloproteinase; GC, gastric cancer; DLT, dose limiting toxicity; DCR, disease control rate; AEs, adverse events; RP2D, recommended Phase II dose; DFS, disease-free survival; DOR, duration of response; ORR, overall response rate; PFS, progression-free survival; OS, overall survival; $m$, months; CSF, colony stimulating factor; chemo, chemotherapy; IDO, indoleamine-2,3-dioxygenase; Neoadj, neoadjuvant; GEJ, gastroesophageal junction; PARP, poly (ADP-ribose) polymerase inhibitor; PCR, pathologic complete response; Cis/5FU, SOX, S-I and oxaliplatin; CapOX, capecitabine and oxaliplatin. 
multiple tumor types. The ongoing Phase I/II CheckMate-032 investigating the safety and efficacy of nivolumab as a single agent or in combination with Ipi in heavily pretreated patients with advanced or metastatic solid tumors, including GC patients (NCT01928394), has been partly reported. ${ }^{52}$ Results from $160 \mathrm{GC}$ patients enrolled in 3 arms nivolumab $3 \mathrm{mg} /$ $\mathrm{kg}$ (N3), nivolumab $1 \mathrm{mg} / \mathrm{kg}+$ Ipi $3 \mathrm{mg} / \mathrm{kg}(\mathrm{N} 1+\mathrm{I} 3)$, and nivolumab $3 \mathrm{mg} / \mathrm{kg}+$ Ipi $1 \mathrm{mg} / \mathrm{kg}(\mathrm{N} 3+\mathrm{I} 1)$ were reported. Eighty-one patients harbored PD-L1-negative tumors while 35 patients harbored tumors with $>1 \%$ PD-L1 expression. Tumor PD-L1 expression was assessed retrospectively in pretreatment tumor specimens with the Dako assay (28-8 pharmDx). Toxicities were more common in the $\mathrm{N} 1+\mathrm{I} 3$ group with $84 \%$ versus N3 (70\%), and N3+I1 (75\%). Common treatment-related adverse events (TRAEs) in all 3 groups included fatigue, pruritus, diarrhea, nausea, and decreased appetite. Thyroid disease was more common in $\mathrm{N} 1+\mathrm{I} 3$ compared to the other groups. Grade 3-4 TRAEs in the N3, $\mathrm{N} 1+\mathrm{I} 3$, and $\mathrm{N} 3+\mathrm{I} 1$ were $17 \%, 45 \%$, and $27 \%$, respectively. Important grade 3-4 TRAEs included pneumonitis, diarrhea, transaminitis, and increased amylase. Among 154 evaluable patients, the confirmed objective response rate (ORR) was 16\%: 14\% (N3), 26\% (N1+I3), and 10\% (N3+I1), including 2 patients with complete response ( 1 in $\mathrm{N} 3 ; 1$ in $\mathrm{N} 1+\mathrm{I} 3$ ) and a disease control rate (DCR; ORR + stable disease [SD]) of $38 \%$. The median OS in the $\mathrm{N} 3, \mathrm{~N} 1+\mathrm{I} 3$, and $\mathrm{N} 3+\mathrm{I} 1$ arms were 5.0 (3.4-12.4), 6.9 (3.6- not accessed [NA]), and 4.8 (3.0-9.1), respectively (Table 2 ). ${ }^{52}$ Up to $44 \%$ patients with PD-L1 expression responded to $\mathrm{N} 1+\mathrm{I} 3$ combination compared with $27 \%$ for the other 2 arms. Despite relative higher toxicity, the $\mathrm{N} 1+\mathrm{I} 3$ combination showed encouraging activity and led to initiation of the CheckMate-649 Phase III trial (NCT02872116). In this study, the investigators plan to enroll 870 patients with untreated advanced or metastatic GC/gastroesophageal junction (GEJ) cancer with or without PD-L1 expression to receive nivolumab + ipi (4 doses; followed by nivolumab monotherapy) versus investigator's choice of capecitabine/oxaliplatin (XELOX) or fluorouracil/ leucovorin/oxaliplatin (FOLFOX).${ }^{53}$ Primary endpoint is OS in patients with PD-L1+ tumors. Secondary endpoints include OS in all patients and progression-free survival (PFS) and time to symptom deterioration in all patients and patients with PD-L1+ tumors. ${ }^{53}$

Preliminary data from a double-blinded, randomized, Phase III trial (ONO-4538/BMS-936558) demonstrated the efficacy of nivolumab as salvage treatment as a third or later line of treatment in 493 patients with advanced gastric or gastroesophageal junction cancer compared to placebo (NCT02267343). ${ }^{54} \mathrm{PD}-1 / \mathrm{L} 1$ positivity was not required for study enrollment. Median OS was 5.32 months with nivolumab versus 4.14 months with placebo (hazard ratio $[\mathrm{HR}], 0.63 ; 95 \%$ confidence interval [CI], 0.50-0.78; $p<0.0001)$. OS rates at 6 and 12 months were $46.4 \%$ versus $34.7 \%$ and $26.6 \%$ versus $10.9 \%$, respectively. Median PFS was longer with nivolumab than placebo (1.61 vs 1.45 months) with HR, $0.60 ; 95 \%$ CI, 0.49-0.75; $p<0.0001$ (Table 2). The ORR was $11.2 \%$ with nivolumab versus $0 \%$ with placebo $(p<0.0001)$. Grade $\geq 3$ drug-related adverse events (AEs) occurred in $11.5 \%$ of nivolumab and $5.5 \%$ of placebo. ${ }^{54}$ Biomarker analysis is under investigation.

\section{Pembrolizumab}

The large multi-cohort Phase Ib KEYNOTE-012 first highlighted the activity of pembrolizumab in PD-L1-positive

Table 2 Clinical activity of PD-I/PD-LI and CTLA-4-directed therapies in advanced gastric cancer

\begin{tabular}{|c|c|c|c|c|c|c|c|c|c|c|c|}
\hline Study & Phase & Trial population & $n$ & $\begin{array}{l}\text { ORR } \\
\text { (\%) }\end{array}$ & $\begin{array}{l}6 \mathrm{~m} \\
\text { PFS } \\
(\%)\end{array}$ & $\begin{array}{l}6 \mathrm{~m} \\
\text { OS } \\
(\%)\end{array}$ & $\begin{array}{l}\text { I2 m } \\
\text { PFS } \\
(\%)\end{array}$ & $\begin{array}{l}12 \mathrm{~m} \\
\text { OS } \\
(\%)\end{array}$ & $\begin{array}{l}\text { Median } \\
\text { OS } \\
(\mathrm{m})\end{array}$ & $\begin{array}{l}\text { Median } \\
\text { PFS } \\
\text { (m) }\end{array}$ & Ref \\
\hline KEYNOTE-0I 2 & $\mathrm{lb}$ & Advanced GC & 39 & 22 & 26 & 66 & NR & 42 & 11.4 & 1.9 & 43 \\
\hline KEYNOTE-028 & $\mathrm{lb}$ & Advanced esophageal & 23 & 30 & 30 & NR & 21.7 & NR & NR & NR & 55 \\
\hline CheckMate-032 & $\mathrm{I} / \mathrm{II}$ & Advanced GC & 59 & 14 & 18 & 49 & 7 & 36 & 5 & 1.3 & 52 \\
\hline \multicolumn{12}{|l|}{ N 3 mg/kg } \\
\hline CheckMate-032 & $\mathrm{I} / \mathrm{II}$ & Advanced GC & 52 & 10 & 9 & 43 & NR & NR & 4.6 & 1.6 & 52 \\
\hline \multicolumn{12}{|c|}{ N 3mg/kg, I I mg/kg } \\
\hline CheckMate-032 & $\mathrm{I} / \mathrm{II}$ & Advanced GC & 49 & 26 & 18 & 54 & 18 & 34 & 6.9 & 1.5 & 52 \\
\hline \multicolumn{12}{|c|}{ N Img/kg, I 3mg/kg } \\
\hline JAVELIN & $\mathrm{lb}$ & Advanced GC/GEJ, second line & 62 & 9.7 & NR & NR & NR & NR & NR & 1.5 & 56 \\
\hline Tremelimumab & ॥ & Advanced GC, esophageal & 18 & 5 & NR & NR & NR & 33 & 4.8 & 2.8 & 73 \\
\hline ONO-4538 & III & $>2$ prior lines, gastric, GEJ & 493 & 11.2 & NR & 46.4 & 7.6 & 26.6 & 5.32 & 1.6 & 54 \\
\hline
\end{tabular}

Notes: Data reflect updates through ASCO GI 20I7. For the JAVELIN study, only the second line subgroup is reported.

Abbreviations: GC, gastric cancer; GEJ, gastroesophageal junction; N, nivolumab; I, ipilimumab; ORR, overall response rate; PFS, progression-free survival; OS, overall survival; m, months; NR, not reported; ASCO, American Society of Clinical Oncology. 
GC (NCT01848834). Of the 162 patients with GC who were assessed for PD-L1 expression, 65 (40\%) had PDL1-positive tumors and 39 were enrolled in the trial. Among the 39 patients with gastric tumors expressing PD-L1 (IHC positive in $>1 \%$ of tumor and/or contiguous mononuclear inflammatory cells using Dako Assay, 22C3 antibody), the majority failed prior treatments (1-5 lines of therapies) and were treated with pembrolizumab at $10 \mathrm{mg} / \mathrm{kg}$ intravenously every 2 weeks. ${ }^{43}$ Treatment with pembroluzumab led to partial response (PR) in 8 patients $(22 \%)$ and SD in 5 patients (13\%) with no significant differences between Asian and non-Asian patients. The median PFS as assessed by central review was 1.9 months $(95 \% \mathrm{CI}, 1.8-3.5)$, with 6-month PFS of $26 \%(95 \%$ CI, 13-41) (Table 2). Median OS was 11.4 months (95\% CI, 5.7-not reached) (Table 2). Common grade 1 or 2 AEs included poor appetite (13\%), fatigue $(13 \%)$, pruritus (13\%), hypothyroidism (4\%), and arthralgia (4\%). This study did not find an association between the interferon- $\gamma$ signature score and overall response $(p=0.070)$ or PFS $(p=0.28) .{ }^{43}$ The interferon- $\gamma$ signature score was calculated as the average of the normalized values of the 6-gene signature (CXCL9, CXCL10, IDO1, IFNG, HLA-DRA, and STAT1). These 6 genes were significantly associated with response to pembrolizumab in melanoma. The similarly designed KEYNOTE-028 (NCT02054806) is a nonrandomized, multi-cohort, Phase Ib trial of pembrolizumab for PD-L1positive advanced solid tumors. The updated results of KEYNOTE-028 esophageal/GEJ cohort included 23 patients with esophageal cancer treated with pembrolizumab at $10 \mathrm{mg} / \mathrm{kg}$ intravenously every 2 weeks up to 2 years. ${ }^{55}$ The majority of patients were Asian ( $n=12)$, and $74 \%$ of all patients had esophageal squamous cell carcinoma $(n=17)$, and progressed on $>2$ lines of therapy $(n=20)$. Nine patients $(39 \%)$ had AEs, most commonly decreased appetite $(n=3,13.0 \%)$. irAEs, regardless of attribution by the investigator, were grade 2 hypothyroidism $(n=2,8.7 \%)$ and adrenal insufficiency $(n=1$, $4.3 \%$ ). Treatment-related events did not lead to any death or study discontinuation. Seven patients had PR (30\%) and 3 patients had SD (13\%). The PFS at 6 and 12 months were $30.4 \%$ and $21.7 \%$, respectively (Table 2 ). Pembrolizumab led to a higher response rate in adenocarcinoma (40\%) than squamous cell histology (29\%). The study also attempted to analyze response rate and duration using the interferon inflammatory 6-gene-expression signature (IDO1, CXCL10, CXCL9, HLA-DRA, STAT1, and IFN- $\gamma$ ). Patients with low signature score (non-inflamed) tumors generally had lower response rates and did not show delays in progression. Conversely, delays in progression and increased ORR tended to occur among patients with higher immune gene signatures scores. Similar patterns were observed for head and neck and GC cohorts.

Although there are multiple caveats in cross-trial comparisons, it is important to note that the KEYNOTE-012 and KEYNOTE-028 studies included a large proportion of patients ( $\sim 60 \%$ in KEYNOTE-012) who had received $\geq 3$ prior lines of therapy, a population in which no large trial has demonstrated significant activity. The relatively small sample sizes limit the ability to determine differential activity of checkpoint inhibitors in relation to the number and type of prior therapies although this remains an interesting question. The ORR and 6-month survivals in these heterogenous populations of pretreated patients approach that of true $2 \mathrm{~L}$ studies, which is encouraging. It should be noted that PFS has limitations in analyzing immunotherapy trials, and landmark analyses such as 6 and 12-month survival may be more reflective of benefit given the long-tail/plateau pattern observed in Kaplan-Meier analyses from multiple other tumor types (Table 2).

\section{Anti-PD-LI antibodies \\ Avelumab}

Avelumab (MSB0010718C) is a fully human anti-PD-L1 IgG1 antibody being investigated in a Phase Ib JAVELIN trial in patients with advanced gastric or GEJ cancers who had at least 1 prior therapy (2L) or who received avelumab as switch maintenance (SwM) after chemotherapy (NCT01772004). ${ }^{56}$ The data from 151 patients ( 62 patients in $2 \mathrm{~L}, 89$ patients in SwM group) receiving $10 \mathrm{mg} / \mathrm{kg}$ avelumab were presented at the American Society of Clinical Oncology 2016 meeting (Chicago, USA). The most common AEs were infusionrelated reactions $(19[12.6 \%])$ and fatigue (16 [10.6\%]). Fifteen patients $(9.9 \%)$ experienced grade $\geq 3$ toxicities, including fatigue, asthenia, thrombocytopenia, anemia, and increased gamma-glutamyl transpeptidase. One patient died of hepatic failure secondary to autoimmune hepatitis. In the $2 \mathrm{~L}$ group, the ORR, DCR, and median PFS were 6/62 patients $(9.7 \%), 29.0 \%$, and 6.0 weeks (95\% CI, 5.7, 6.4), respectively. In the SwM group, the ORR, DCR, and median PFS were 8/89 patients (9\%), 57.3\%, and 12.0 weeks $(95 \%$ CI, 9.9, 17.6), respectively (Table 2). In this study, PD-L1 expression status is defined as positive or negative using the Dako IHC assay (anti-PD-L1 clone 73-10, Merck KGaA) at various cutoff levels based on the quanity and intensity of staining. These cutoff levels included at least $1 \%, 5 \%$, or $25 \%$ TCs or at least $10 \%$ tumor-infiltrating ICs. Among 22 patients in the $2 \mathrm{~L}$ groups who were evaluated for PD-L1 expression, 
the ORR in the PD-L1-positive subset was higher than in patients with PD-L1-negative tumors (18.2\% vs 9.1\%). In the SwM group $(n=52)$, patients with tumors harboring PD-L1 expression also exhibited higher responses than tumors without PD-L1 expression (10\% vs 3.1\%). ${ }^{56}$

\section{Durvalumab (MEDI4736)}

Durvalumab (AstraZeneca, London, UK) is an engineered human anti-PD-L1 IgG1 isotype that binds PD-L1, preventing its binding to PD-1 and CD80. In a Phase I study, patients with solid tumors including GC (NCT01693562) patients were treated with durvalumab up to $10 \mathrm{mg} / \mathrm{kg}$ intravenously every 2 weeks for up to 12 months. ${ }^{57}$ Treatment-related AEs occurred in $33 \%$ of patients, including $7 \%$ patients with grade 3 toxicities. The most frequently observed treatmentrelated AEs were fatigue (13\%), nausea $(8 \%)$, rash $(6 \%)$, vomiting (5\%), and pyrexia (5\%). Preliminary efficacy data demonstrated an ORR of $25 \%$ in (4/16) patients with GC. Two cases of heavily pretreated GC remained stable over 24 weeks, exceeding the current median PFS of approved 2L therapies. ${ }^{57}$

In summary, checkpoint inhibitors demonstrated clinical benefit in patients with advanced and refractory GC. As observed in other tumor types, PD-L1 expression seems to be associated with higher response to checkpoint inhibitors. In the KEYNOTE-012 trial, the ORR, 12-month OS, and median OS among patients with PD-L1-positive tumors who received pembrolizumab were $22 \%, 42 \%$, and 11.4 months, respectively. The proportion of patients who had 0 , $1,2,3$, and $\geq 4$ lines of therapies were $15 \%, 18 \%, 10 \%, 21 \%$, and $36 \%$, respectively. In contrast, the single agent activity of nivolumab in CheckMate-032 yieled ORR of 14\% and median OS of 5 months in PD-1/L1 unselected advanced GC/ GEJ patients who failed previous treatments ( $41 \%$ of patients had 1 and 56\% had 2-3 lines of prior treatments). The lower ORR and median OS in this trial may be due to the inclusion of unselected patients. However, these 2 studies included different patient populations, and one cannot conclude that activity is equivalent based on these limited data sets. The addition of Ipi (3 $\mathrm{mg} / \mathrm{kg})$ to nivolumab ( $1 \mathrm{mg} / \mathrm{kg}$ ) yielded an impressive response rate (ORR of $26 \%$ in unselected patients and $44 \%$ in patients with PD-L1 expression) at the expense of higher incidence of TRAEs. This combination is promising in patients with good performance status and low combordity score, and Phase III trials are ongoing (Table 2, NCT02872116). Longer follow-up is required to determine the durability of response from this combination. Data from ONO-4538 also showed similar single agent activity of nivolumab in unselected patients with advanced and heavily pretreated GC ( $>2$ lines of therapy) compared with the CheckMate-032 study. There are currently insufficient data to determine differences in clinical efficacy between PD-1 and PD-L1 targeting approaches.

\section{Combination therapy}

The efficacy of anti-PD-1-directed therapy in GC has spawned combination studies with other active targeted biologic agents. Preclinical work has shown synergistic activity between blockade of both the PD-1/PD-L1 axis and the vascular endothelial growth factor (VEGF)/vascular endothelial growth factor receptor (VEGFR) pathway. ${ }^{58}$ Dual inhibition has the potential to induce deeper responses, longer PFS, and ultimately prolonged OS. Early phase studies of combination PD-1/PD-L1 and VEGF/VEGFR blockade in other solid tumors have already shown an acceptable safety profile but outcomes data are yet to be established. ${ }^{59-62}$ Orthogonal chemo-immuno combination support from NSCLC was recently reported. The randomized, open-label, Phase II KEYNOTE-021 trial demonstrated that the addition of pembrolizumab to carboplatin and pemetrexed yielded higher response rate compared to carboplatin and pemetrexed alone $(55 \%$ vs $29 \%, p=0.0016)$ in patients with treatmentnaïve NSCLC (NCT02039674). ${ }^{62}$ The incidence of grade 3 or worse TRAEs was similar between the 2 arms, which consisted mainly of neutropenia, anemia, thrombocytopenia, and fatigue. The positive result from this study is being further explored in an ongoing randomized, double-blind, Phase III KEYNOTE-189 trial (NCT02578680).

In GC, a Phase Ia/Ib study of combination anti-PD-L1 and anti-VEGFR2 antibodies, durvalumab, and ramucirumab has been investigated and the interim safety and efficacy data of 40 patients with refractory GC/GEJ tumors have been presented (NCT02443324). ${ }^{63}$ In this study, PD-L1 expression $>1 \%$ was classified as positive although not an inclusion criterion for study entry. The dosing regimen was pembrolizumab $200 \mathrm{mg}$ on day 1 every 3 weeks and ramurcirumab $8 \mathrm{mg} / \mathrm{kg}$ on days 1 and 8 (cohort A) or $10 \mathrm{mg} / \mathrm{kg}$ on day 1 only (cohort B). Preliminary efficacy data showed 3 of $40(7.5 \%)$ patients (PD-L1 negative, $\mathrm{n}=1$; PD-L1 positive, $\mathrm{n}=2$ ) responded to treatment with a 45\% DCR. Median PFS was 2.10 months $(95 \%$ CI, 1.18-4.04) and 2.60 months (1.38, not reported) for cohorts $\mathrm{A}$ and $\mathrm{B}$, respectively. Common toxicities included fatigue $(30 \%)$, infusion-related reaction (12.5\%), decreased appetite (12.5\%), pruritus $(10 \%)$, maculopapular rash (10\%), and hypertension $(10 \%)$. Ten $(25 \%)$ patients had grade $3-4$ toxicities, most commonly colitis $(7.5 \%)$ and hypertension $(7.5 \%) .{ }^{63}$ 
Combination anti-PD-1 and chemotherapy approaches have also undergone active investigation given the recognition that chemotherapy may also promote immunogenic cell death. ${ }^{64}$ In the ongoing Phase II KEYNOTE-059 study, preliminary data were obtained from 25 patients with advanced HER2-negative gastric adenocarcinoma who were treated with first-line 5-fluorouracil and cisplatin in combination with pembrolizumab. All 25 patients experienced TRAEs of any grade, and 72\% (18/25) experienced grade 3 and 4 TRAEs. No patients were discontinued due to pembrolizumab-related AEs suggesting the combination has a manageable side effect profile. ${ }^{65,66}$ The ongoing Phase III KEYNOTE-062 study (NCT02494583) is carrying this combination forward randomizing advanced, metastatic gastric/GEJ adenocarcinoma patients whose tumors express PD-L1 to pembrolizumab monotherapy versus pembrolizumab plus cisplatin and fluorouracil versus placebo plus cisplatin and fluorouracil for treatment-naïve diseases (Table 2). The promising results of such anti-PD-1/PD-L1 single agent and combination studies in advanced disease have naturally spawned multiple trials in earlier stage disease, including the adjuvant nivolumab Phase III trial in resected esophageal and GEJ (CheckMate-577), and a Phase I neoadjuvant trial of nivolumab and Ipi in stage II-III patients (NCT03044613). ${ }^{53}$

Given that patients with HER2-overexpressing tumors invariably demonstrate progression and resistance to trastuzumab-containing therapies, combination efforts are also investigating combining PD-1 blockade with anti-HER2 agents. Preclinical studies indicate that Her-2 inhibition can promote T-cell activation and trafficking and boost natural killer cell secretion of interferon- $\gamma$ and antibody-dependent cellular toxicity which may be prime for PD-1/L1 effectiveness. ${ }^{67}$ A Phase Ib/II, open-label, dose-escalation study is investigating the novel anti-HER2 mAb margetuximab in combination with pembrolizumab in patients with advanced HER2-amplified GC who are refractory to standard trastuzumab-based combination chemotherapy (NCT02689284) (Table 1). ${ }^{68}$ Dual targeting of both the tumor microenvironment as well as multiple nodes in the cancer immunity cycle is also an attractive option. These include combining antiPD-1/PD-L1 therapies with agents inhibiting other immune checkpoints (TIM3, LAG3), T-cell costimulatory agonist antibodies (GITR, OX40, 4-1BB), enzymatic inhibitors (IDO-1), as well as radiation and other cytotoxic drugs. Additionally, the combination of nivolumab and GS-5745, a matrix metalloproteinase 9 inhibitor, is also being investigated in patients with unresectable or recurrent GC/GEJ adenocarcinoma (NCT02864381). These trials are all under ongoing clinical investigation (Table 1). Notably, there is a paucity of GC-specific preclinical data providing specific combinatorial rationale. Radiation therapy, however, has been of significant interest in PD-1/PD-L1 combinatorial approaches due to the well annotated although rarely observed clinical phenomenon of the abscopal response in cancer radiotherapy, in which nonirradiated metastatic lesions are noted to regress after radiation to the primary tumor site. In multiple preclinical tumor models, combination radiotherapy and PD-1/PD-L1 axis blockade exhibit synergistic antitumor activity and reduce tumor-infiltrating myeloid-derived suppressor cells. ${ }^{69,70}$ GC-specific trials are ongoing including studies combining pembrolizumab with palliative radiotherapy in the metastatic setting, as well as with neoadjuvant chemoradiotherapy for GEJ and gastric cardia cancers in earlier stage resectable disease (NCT02730546). ${ }^{71}$

\section{Future directions}

Early trials involving immunotherapy in non-GC tumors were notable for prolonged responses in some patients, but often low ORRs highlighting the need for response biomarkers. ${ }^{11,13-15}$ Among the reported trials, there is a general trend toward higher ORR in PD-1 and/or PD-L1-positive samples (TCs or ICs). Among the subgroup of MSI-H tumors, it seems clear that high TMB is the biologic underpinning of the observed high response rates. Results from multiple ongoing trials in GC will further refine the roles for biomarkers selection and optimal combination approaches.

Importantly, $\sim 40 \%$ of patients with GC develop peritoneal carcinomatosis and ascites, a phenotype associated with a particularly poor prognosis. ${ }^{72}$ More work is needed to study the immune milieu of the peritoneum in these patients with readily accessible tissue and stromal cells. Activity of checkpoint inhibitors has not been reported specifically in this population and perhaps intraperitoneal delivery of single agent or rational combinations can be considered.

This study has reviewed the role of PD-1 in GC with a focus on emerging clinical data. With the expected approval of PD-1 inhibitors, many important questions remain; however, promising results are expected for the future of GC.

\section{Acknowledgments}

The authors would like to acknowledge important contributions from authors whose works could not be cited due to space constraints. Efforts in manuscript preparation for Dr Joseph Chao were supported by National Institutes of Health grant 5K12CA001727-22. The content is solely the responsibility of the authors and does not necessarily represent the official views of the National Institutes of Health. 


\section{Disclosure}

Joseph Chao has received research funding from Merck in support of an investigator-initiated clinical trial (NCT02830594).

The authors report no other conflicts of interest in this work.

\section{References}

1. Torre LA, Bray F, Siegel RL, Ferlay J, Lortet-Tieulent J, Jemal A. Global cancer statistics, 2012. CA Cancer J Clin. 2015;65(2):87-108.

2. Siegel RL, Miller KD, Jemal A. Cancer statistics, 2015. CA Cancer J Clin. 2015;65(1):5-29.

3. Bang Y-J, Van Cutsem E, Feyereislova A, et al. Trastuzumab in combination with chemotherapy versus chemotherapy alone for treatment of HER2-positive advanced gastric or gastro-oesophageal junction cancer (ToGA): a phase 3, open-label, randomised controlled trial. Lancet. 2010;376(9742):687-697.

4. Wilke H, Muro K, Van Cutsem E, et al. Ramucirumab plus paclitaxel versus placebo plus paclitaxel in patients with previously treated advanced gastric or gastro-oesophageal junction adenocarcinoma (RAINBOW): a double-blind, randomised phase 3 trial. Lancet Oncol. 2014;15(11):1224-1235.

5. Fuchs CS, Tomasek J, Yong CJ, et al. Ramucirumab monotherapy for previously treated advanced gastric or gastro-oesophageal junction adenocarcinoma (REGARD): an international, randomised, multicentre, placebo-controlled, phase 3 trial. Lancet. 2014;383(9911):31-39.

6. Yang W, Raufi A, Klempner SJ. Targeted therapy for gastric cancer: molecular pathways and ongoing investigations. Biochim Biophys Acta. 2014;1846(1):232-237.

7. Raufi AG, Klempner SJ. Immunotherapy for advanced gastric and esophageal cancer: preclinical rationale and ongoing clinical investigations. J Gastrointest Oncol. 2015;6(5):561.

8. Chow LQ, Haddad R, Gupta S, et al. Antitumor activity of pembrolizumab in biomarker-unselected patients with recurrent and/or metastatic head and neck squamous cell carcinoma: results from the phase Ib KEYNOTE-012 expansion cohort. J Clin Oncol. 2016;34(32):3838-3845.

9. Ansell SM, Lesokhin AM, Borrello I, et al. PD-1 blockade with nivolumab in relapsed or refractory Hodgkin's lymphoma. $N$ Engl $J$ Med. 2015;372(4):311-319.

10. Motzer RJ, Escudier B, McDermott DF, et al. Nivolumab versus everolimus in advanced renal-cell carcinoma. NEngl JMed. 2015;373(19):1803-1813.

11. Rosenberg JE, Hoffman-Censits J, Powles T, et al. Atezolizumab in patients with locally advanced and metastatic urothelial carcinoma who have progressed following treatment with platinum-based chemotherapy: a singlearm, multicentre, phase 2 trial. Lancet. 2016;387(10031):1909-1920.

12. Fehrenbacher L, Spira A, Ballinger M, et al. Atezolizumab versus docetaxel for patients with previously treated non-small-cell lung cancer (POPLAR): a multicentre, open-label, phase 2 randomised controlled trial. Lancet. 2016;387(10030):1837-1846.

13. Borghaei H, Paz-Ares L, Horn L, et al. Nivolumab versus docetaxel in advanced nonsquamous non-small-cell lung cancer. $N$ Engl J Med. 2015;373(17):1627-1639.

14. Brahmer J, Reckamp KL, Baas P, et al. Nivolumab versus docetaxel in advanced squamous-cell non-small-cell lung cancer. $N$ Engl J Med. 2015;373(2):123-135.

15. Herbst RS, Baas P, Kim D-W, et al. Pembrolizumab versus docetaxel for previously treated, PD-L1-positive, advanced non-small-cell lung cancer (KEYNOTE-010): a randomised controlled trial. Lancet. 2016;387(10027):1540-1550.

16. Garon EB, Rizvi NA, Hui R, et al. Pembrolizumab for the treatment of non-small-cell lung cancer. N Engl J Med. 2015;372(21):2018-2028.

17. Larkin J, Chiarion-Sileni V, Gonzalez R, et al. Combined nivolumab and ipilimumab or monotherapy in untreated melanoma. NEngl J Med. 2015;2015(373):23-34.

18. Ribas A, Puzanov I, Dummer R, et al. Pembrolizumab versus investigator-choice chemotherapy for ipilimumab-refractory melanoma (KEYNOTE-002): a randomised, controlled, phase 2 trial. Lancet Oncol. 2015;16(8):908-918.
19. Rooney MS, Shukla SA, Wu CJ, Getz G, Hacohen N. Molecular and genetic properties of tumors associated with local immune cytolytic activity. Cell. 2015;160(1-2):48-61.

20. Chen DS, Mellman I. Oncology meets immunology: the cancerimmunity cycle. Immunity. 2013;39(1):1-10.

21. Le DT, Uram JN, Wang H, et al. PD-1 blockade in tumors with mismatch-repair deficiency. $N$ Engl J Med. 2015;372(26): 2509-2520.

22. Kaneda MM, Messer KS, Ralainirina N, et al. PI3K $\gamma$ is a molecular switch that controls immune suppression. Nature. 2016;539(7629): 437-442.

23. Cancer Genome Atlas Research Network. Comprehensive molecular characterization of gastric adenocarcinoma. Nature. 2014;513(7517):202-209.

24. Cancer Genome Atlas Research Network. Comprehensive molecular characterization of human colon and rectal cancer. Nature. 2012;487(7407):330-337.

25. Böger C, Behrens H-M, Mathiak M, Krüger S, Kalthoff H, Röcken C. PD-L1 is an independent prognostic predictor in gastric cancer of Western patients. Oncotarget. 2016;7(17):24269-24283.

26. Bernal M, Concha A, Sáenz-López P, et al. Leukocyte infiltrate in gastrointestinal adenocarcinomas is strongly associated with tumor microsatellite instability but not with tumor immunogenicity. Cancer Immunol Immunother. 2011;60(6):869-882.

27. Spranger S, Gajewski TF. Tumor-intrinsic oncogene pathways mediating immune avoidance. Oncoimmunology. 2016;5(3):e1086862.

28. Gajewski TF. The next hurdle in cancer immunotherapy: overcoming the non-T-cell-inflamed tumor microenvironment. Semin Oncol. 2015;42(4):663-671.

29. Hu-Lieskovan S, Ribas A. New combination strategies using programmed cell death 1/programmed cell death ligand 1 checkpoint inhibitors as a backbone. Cancer J. 2017;23(1):10-22.

30. Blank CU, Haanen JB, Ribas A, Schumacher TN. Cancer immunology. The "cancer immunogram". Science. 2016;352(6286):658-660.

31. Spranger S, Bao R, Gajewski TF. Melanoma-intrinsic $\beta$-catenin signalling prevents anti-tumour immunity. Nature. 2015;523(7559): 231-235.

32. Lin SJ, Gagnon-Bartsch JA, Tan IB, et al. Signatures of tumour immunity distinguish Asian and non-Asian gastric adenocarcinomas. Gut. 2015;64(11):1721-1731.

33. Rizvi NA, Hellmann MD, Snyder A, et al. Mutational landscape determines sensitivity to PD-1 blockade in non-small cell lung cancer. Science. 2015;348(6230):124-128.

34. Ribic CM, Sargent DJ, Moore MJ, et al. Tumor microsatellite-instability status as a predictor of benefit from fluorouracil-based adjuvant chemotherapy for colon cancer. N Engl J Med. 2003;349(3):247-257.

35. Lesokhin AM, Callahan MK, Postow MA, Wolchok JD. On being less tolerant: enhanced cancer immunosurveillance enabled by targeting checkpoints and agonists of T cell activation. Sci Transl Med. 2015;7(280):280sr1.

36. Rosenberg JE, Hoffman-Censits J, Powles T, et al. Atezolizumab in patients with locally advanced and metastatic urothelial carcinoma who have progressed following treatment with platinum-based chemotherapy: a single-arm, multicentre, phase 2 trial. Lancet. 2016;387(10031):1909-1920.

37. Balar AV, Galsky MD, Rosenberg JE, et al. Atezolizumab as first-line treatment in cisplatin-ineligible patients with locally advanced and metastatic urothelial carcinoma: a single-arm, multicentre, phase 2 trial. Lancet. 2017;389(10064):67-76.

38. McGranahan N, Furness AJ, Rosenthal R, et al. Clonal neoantigens elicit $\mathrm{T}$ cell immunoreactivity and sensitivity to immune checkpoint blockade. Science. 2016;351(6280):1463-1469.

39. Reck M, Rodriguez-Abreu D, Robinson AG, et al. Pembrolizumab versus chemotherapy for PD-L1-positive non-small-cell lung cancer. N Engl J Med. 2016;375(19):1823-1833.

40. Cho J, Lee J, Bang H, et al. Programmed cell death-ligand 1 expression predicts survival in patients with gastric carcinoma with microsatellite instability. Oncotarget. 2017;8(8):13320-13328. 
41. Kim JW, Nam KH, Ahn SH, et al. Prognostic implications of immunosuppressive protein expression in tumors as well as immune cell infiltration within the tumor microenvironment in gastric cancer. Gastric Cancer. 2016;19(1):42-52.

42. Wu C, Zhu Y, Jiang J, Zhao J, Zhang X-G, Xu N. Immunohistochemical localization of programmed death-1 ligand-1 (PD-L1) in gastric carcinoma and its clinical significance. Acta Histochem. 2006;108(1):19-24.

43. Muro K, Chung HC, Shankaran V, et al. Pembrolizumab for patients with PD-L1-positive advanced gastric cancer (KEYNOTE-012): a multicentre, open-label, phase 1b trial. Lancet Oncol. 2016;17(6):717-726.

44. Gaule P, Smithy JW, Toki M, et al. A quantitative comparison of antibodies to programmed cell death 1 ligand 1. JAMA Oncol. Epub 2016 Aug 18.

45. Scheel AH, Dietel M, Heukamp LC, et al. Harmonized PD-L1 immunohistochemistry for pulmonary squamous-cell and adenocarcinomas. Mod Pathol. 2016;29(10):1165-1172.

46. Sun J, Xu K, Wu C, et al. PD-L1 expression analysis in gastric carcinoma tissue and blocking of tumor-associated PD-L1 signaling by two functional monoclonal antibodies. Tissue Antigens. 2007;69(1):19-27.

47. Eto S, Yoshikawa K, Nishi M, et al. Programmed cell death protein 1 expression is an independent prognostic factor in gastric cancer after curative resection. Gastric Cancer. 2016;19(2):466-471.

48. Schlößer HA, Drebber U, Kloth M, et al. Immune checkpoints programmed death 1 ligand 1 and cytotoxic $\mathrm{T}$ lymphocyte associated molecule 4 in gastric adenocarcinoma. Oncoimmunology. 2016;5(5):e1100789.

49. Xiaofang X, Li Z, Wang J, et al. Analysis of PDL1 expression and T cells infiltration in 1014 gastric cancer patients. J Clin Oncol. 2017;35(suppl 4S; abstract 50).

50. Tanaka H, Tamura T, Hiramatsu S, et al. Role of systemic immuneinflammatory index, tumor infiltrating neutrophils and PD-1+ T cells to predict the postoperative recurrence after $\mathrm{S}-1$ adjuvant chemotherapy for gastric cancer: a retrospective study. J Clin Oncol. 2017;35(suppl 4S; abstract 74)

51. Jiang $\mathrm{Y}$, Zhang $\mathrm{Q}, \mathrm{Hu} \mathrm{Y}$, et al. ImmunoScore signature: a prognostic and predictive tool in gastric cancer. Ann Surg. Epub 2016 Dec 20.

52. Janjigian YY, Bendell J, Calvo E, et al. CheckMate-032: phase I/II, open-label study of safety and activity of nivolumab (nivo) alone or with ipilimumab (ipi) in advanced and metastatic (A/M) gastric cancer (GC). J Clin Oncol. 2016;34(15 Suppl):4010.

53. Janjigian YY, Adenis A, Aucoin J-S, et al. Checkmate 649: a randomized, multicenter, open-label, phase 3 study of nivolumab (Nivo) plus ipilimumab (Ipi) versus oxaliplatin plus fluoropyrimidine in patients (Pts) with previously untreated advanced or metastatic gastric $(\mathrm{G})$ or gastroesophageal junction (GEJ) cancer. J Clin Oncol. 2017;35.

54. Kang Yoon-Koo, Satoh Taroh, Ryu Min-Hee, et al. Nivolumab (ONO4538/BMS-936558) as salvage treatment after second or later-line chemotherapy for advanced gastric or gastro-esophageal junction cancer (AGC): a double-blinded, randomized, phase III trial. J Clin Oncol. 2017;35(suppl 4S; abstract 2).

55. Doi T, Piha-Paul SA, Jalal SI, et al. Updated results for the advanced esophageal carcinoma cohort of the phase Ib KEYNOTE-028 study of pembrolizumab (MK-3475). J Clin Oncol. 2016;34(suppl 4S; abstr 7).

56. Chung HC, Arkenau H-T, Wyrwicz L, et al. Avelumab (MSB0010718C; anti-PD-L1) in patients with advanced gastric or gastroesophageal junction cancer from JAVELIN solid tumor phase Ib trial: analysis of safety and clinical activity. J Clin Oncol. 2016;34(suppl; abstr 4009).

57. Segal NH, Antonia SJ, Brahmer JR, et al. Preliminary data from a multi-arm expansion study of MEDI4736, an anti-PD-L1 antibody. J Clin Oncol. 2014;32:5s.
58. Voron T, Marcheteau E, Pernot S, et al. Control of the immune response by pro-angiogenic factors. Front Oncol. 2014;4:70.

59. Reardon DA, De Groot JF, Colman H, et al. Safety of pembrolizumab in combination with bevacizumab in recurrent glioblastoma (rGBM). $J$ Clin Oncol. 2016;34(suppl; abstr 2010).

60. Dudek AZ, Sica RA, Sidani A, et al. Phase Ib study of pembrolizumab in combination with bevacizumab for the treatment of metastatic renal cell carcinoma: Big Ten Cancer Research Consortium BTCRC-GU14-003. J Clin Oncol. 2016;34(suppl 2S; abstr 559).

61. Solmaz S, Johnstone PA, Forsyth PA, et al. Safety and antitumor activity of hypofractionated stereotactic irradiation (HFSRT) with pembrolizumab (Pembro) and bevacizumab (Bev) in patients (pts) with recurrent high grade gliomas: preliminary results from phase I study. $J$ Clin Oncol. 2016;34(suppl; abstr 2041).

62. Langer CJ, Gadgeel SM, Borghaei H, et al. Carboplatin and pemetrexed with or without pembrolizumab for advanced, non-squamous nonsmall-cell lung cancer: a randomised, phase 2 cohort of the open-label KEYNOTE-021 study. Lancet Oncol. 2016;17(11):1497-1508.

63. Ian Chau JCB, Emiliano Calvo, Rafael Santana-Davila, et al. Interim safety and clinical activity in patients (pts) with advanced gastric or gastroesophageal junction (G/GEJ) adenocarcinoma from a multicohort phase 1 study of ramucirumab (R) plus pembrolizumab (P). JClin Oncol. 2017;35(suppl 4S; abstract 102).

64. Emens LA, Middleton G. The interplay of immunotherapy and chemotherapy: harnessing potential synergies. Cancer Immunol Res. 2015;3(5):436-443.

65. Fuchs CS, Ohtsu A, Tabernero J, et al. Preliminary safety data from KEYNOTE-059: pembrolizumab plus 5-fluorouracil (5-FU) and cisplatin for first-line treatment of advanced gastric cancer. J Clin Oncol. 2016;34(suppl; abstr 4037).

66. Herbst RS, Bendell JC, Isambert N, et al. A phase 1 study of ramucirumab (R) plus pembrolizumab (P) in patients (pts) with advanced gastric or gastroesophageal junction (G/GEJ) adenocarcinoma, nonsmall cell lung cancer (NSCLC), or urothelial carcinoma (UC): phase 1a results. J Clin Oncol. 2016;34(suppl; abstr 3056).

67. Vanneman M, Dranoff G. Combining immunotherapy and targeted therapies in cancer treatment. Nat Rev Cancer. 2012;12(4):237-251.

68. Catenacci DVT, Kim SS, Gold PJ, et al. A phase 1b/2, open label, doseescalation study of margetuximab (M) in combination with pembrolizumab (P) in patients with relapsed/refractory advanced HER2 + gastroesophageal (GEJ) junction or gastric (G) cancer. J Clin Oncol. 2017;35(suppl 4S; abstract TPS219).

69. Vatner RE, Cooper BT, Vanpouille-Box C, Demaria S, Formenti SC. Combinations of immunotherapy and radiation in cancer therapy. Front Oncol. 2014;4:325.

70. Dovedi SJ, Adlard AL, Lipowska-Bhalla G, et al. Acquired resistance to fractionated radiotherapy can be overcome by concurrent PD-L1 blockade. Cancer Res. 2014;74(19):5458-5468.

71. Chao J, Chen Y-J, Frankel PH, et al. Combining pembrolizumab and palliative radiotherapy in gastroesophageal cancer to enhance antitumor T-cell response and augment the abscopal effect. J Clin Oncol. 2017;35(suppl 4S; abstract TPS220).

72. Ikoma N, Blum M, Chiang YJ, et al. Yield of staging laparoscopy and lavage cytology for radiologically occult peritoneal carcinomatosis of gastric cancer. Ann Surg Oncol. 2016;23(13):4332-4337.

73. Ralph C, Elkord E, Burt DJ, et al. Modulation of lymphocyte regulation for cancer therapy: a phase II trial of tremelimumab in advanced gastric and esophageal adenocarcinoma. Clin Cancer Res. 2010;16(5):1662-1672. 
Gastrointestinal Cancer: Targets and Therapy is an international, peer-reviewed, open access journal focusing on gastro-intestinal cancer research, identification of therapeutic targets and the optimal use of preventative and integrated treatment interventions to achieve improved outcomes, enhanced survival and quality of life for the cancer patient. The manuscript management system is completely online and includes a very quick and fair peer-review system. Visit $\mathrm{http}: / /$ www.dovepress.com/testimonials.php to read real quotes from published authors. 